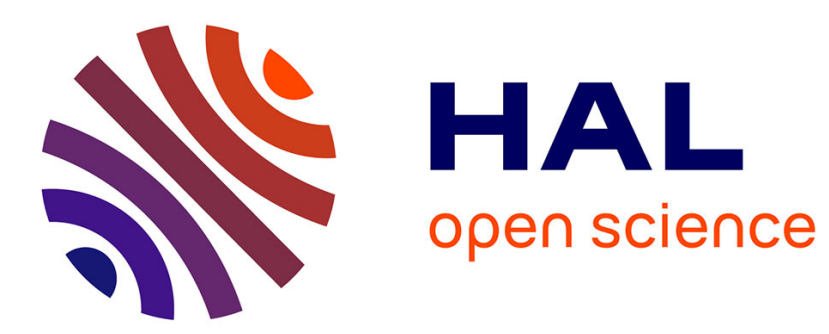

\title{
Stability of quasi-static crack evolution through dimensional reduction
}

Jean-François Babadjian

\section{To cite this version:}

Jean-François Babadjian. Stability of quasi-static crack evolution through dimensional reduction. IUTAM symposium on variational concepts with applications to the mechanics of materials, Sep 2008, Bochum, Germany. pp.1-13, 10.1007/978-90-481-9195-6_1 . hal-00797538

\section{HAL Id: hal-00797538 \\ https://hal.science/hal-00797538}

Submitted on 6 Mar 2013

HAL is a multi-disciplinary open access archive for the deposit and dissemination of scientific research documents, whether they are published or not. The documents may come from teaching and research institutions in France or abroad, or from public or private research centers.
L'archive ouverte pluridisciplinaire HAL, est destinée au dépôt et à la diffusion de documents scientifiques de niveau recherche, publiés ou non, émanant des établissements d'enseignement et de recherche français ou étrangers, des laboratoires publics ou privés. 


\title{
Stability of Quasi-Static Crack Evolution through Dimensional Reduction
}

\author{
Jean-François Babadjian
}

\begin{abstract}
This paper deals with quasi-static crack growth in thin films. We show that, when the thickness of the film tends to zero, any three-dimensional quasi-static crack evolution converges to a two-dimensional one, in a sense related to the $\Gamma$ convergence of the associated total energy. We extend the prior analysis of [2] by adding conservative body and surface forces which allow us to remove the boundedness assumption on the deformation field.
\end{abstract}

\section{Introduction}

In this paper, we study the evolution of cracks in thin structures in a quasi-static setting. Our approach of fracture mechanics is based on a variational model proposed in [10] (see also the monograph [4]) where the (quasi-static) evolution results from the competition - at each time - between a bulk and a surface energy, under a growth constraint on the crack. Many existence results have been obtained (see e.g. $[8,9,11]$ and references therein).

Sometimes a small parameter is involved in the model, and it is an interesting question to study the asymptotic behavior of the model when the parameter tends to zero (see e.g. [14] for the homogenization and [2] for the dimension reduction of quasi-static crack evolution). When dealing static problems, the notion of $\Gamma$-convergence (see [7]) has proven to be a powerful tool to capture the asymptotic behavior of minimizers, or even minimizing sequences. It turns out that even in the quasi-static case, one can define a notion of convergence related to the $\Gamma$ convergence of the associated total energy (see [16] for an abstract theory in the more general framework of rate independent processes).

Jean-François Babadjian

Centre de Mathématiques Appliquées, Ecole Polytechnique, 91128 Palaiseau Cedex, France;

E-mail: babadjian@cmap.polytechnique.fr 
We present here an extension of the result in [2] on the convergence of a quasistatic crack evolution in thin films, as the thickness tends to zero. In [2], an empirical $L^{\infty}$ bound was done on the deformation field (as in [9]) in order to gain compactness in the space $S B V^{p}$ of special functions of bounded variation. It is sometimes possible to justify this assumption as in the antiplanar case (see [11] when $N=2$ ) where it follows from a consequence of the maximum principle. Unfortunately, in the full three-dimensional elasticity, the maximum principle does not hold anymore. We propose here to remove this hypothesis adding suitable conservative bulk and surface forces as in [8]. The price to pay is that the deformation field in not anymore compact in $S B V^{p}$ but in a larger subspace $G S B V_{q}^{p}$ of generalized special functions of bounded variation. The arguments we use in the present paper are very close to those of [2], and for this reason we will only mention the main differences without giving the precise proofs of the results.

The paper is organized as follows: in Section 2, we will describe the model in the physical configuration and state the existence result of [8]. Then, in Section 3, we will reformulate the problem on a rescaled configuration in order to work on a fixed domain. In Section 4, we will perform an asymptotic analysis of the total energy of the system in a static setting, thanks to a $\Gamma$-convergence method. Finally, we will address the asymptotic of the quasi-static problem in Section 5, proving that it converges to a quasi-static evolution associated to the $\Gamma$-limit model.

\section{Description of the Model}

We consider a homogeneous thin film occupying in its reference configuration the cylinder $\Omega^{\varepsilon}:=\omega \times(-\varepsilon, \varepsilon)$, where $\varepsilon>0$ and $\omega$ is a bounded open subset of $\mathbf{R}^{2}$ with Lipschitz boundary. The Dirichlet part of the boundary where the deformation is prescribed is the lateral boundary $\partial_{D} \Omega^{\varepsilon}:=\partial \omega \times(-\varepsilon, \varepsilon)$, while the Neumann part $\partial_{N} \Omega^{\varepsilon}=\omega \times\{-\varepsilon, \varepsilon\}$ is made of the lower and upper sections.

On the lateral boundary $\partial_{D} \Omega^{\varepsilon}$, we impose a time dependent boundary deformation $\phi^{\varepsilon}(t)$ on a finite time interval $[0, T]$, where

$$
t \mapsto \phi^{\varepsilon}(t) \in W^{1,1}\left([0, T] ; W^{1, p}\left(\Omega^{\varepsilon} ; \mathbf{R}^{3}\right) \cap L^{q}\left(\Omega^{\varepsilon} ; \mathbf{R}^{3}\right)\right),
$$

for some $p>1$ and $q \geq 1$.

On the remaining part of the boundary $\partial_{N} \Omega^{\varepsilon}$, we impose a time dependent surface conservative force which will be described in Section 2.3.2.

\subsection{Admissible Cracks}

We fix an open subset $\Omega_{B}^{\varepsilon}$ of $\Omega^{\varepsilon}$ of the form $\Omega_{B}^{\varepsilon}:=\omega \times(-\varepsilon+\varepsilon \eta, \varepsilon-\varepsilon \eta)$ for some $\eta \in(0,1)$, to that the set $\overline{\Omega_{B}^{\varepsilon}}$ represents the brittle part of the body. The set of all 
admissible cracks is given by

$$
\mathcal{R}\left(\overline{\Omega_{B}^{\varepsilon}}\right):=\left\{K: K \text { is rectifiable, } K \widetilde{\subset} \overline{\Omega_{B}^{\varepsilon}} \text { and } \mathscr{H}^{2}(K)<+\infty\right\} .
$$

Note that any admissible crack must lie far enough from the upper and lower sections. The safety region $\Omega^{\varepsilon} \backslash \overline{\Omega_{B}^{\varepsilon}}$ can be interpreted as a layer of unbreakable material (see [8, remark 3.8]).

We denote by $\mathscr{H}^{N-1}$ the $(N-1)$-dimensional Hausdorff measure in $\mathbf{R}^{N}$ (we shall only consider the cases $N=2$ or 3 ), and by $\widetilde{\subset}$ (resp. $\cong$ ) inclusion (resp. equality) up to a set of zero $\mathscr{H}^{N-1}$-measure.

We assume that the energy spent to produce a crack $K$ is of Griffith type, i.e.,

$$
\mathcal{K}(\varepsilon)(K):=\mathscr{H}^{2}(K) .
$$

\subsection{Admissible Deformations}

We refer to [1] for the usual definitions and results on geometric measure theory, $B V, S B V$ and $G S B V$ spaces. Precise definitions of the jump set $S_{u}$ and of the approximate gradient $\nabla u$ of a function $u \in G S B V\left(U ; \mathbf{R}^{d}\right)$, where $U$ is an open subset of $\mathbf{R}^{N}$, can be found in that reference. Following Dal Maso et al. [8], we further define for $p>1$

$$
\begin{aligned}
G S B V^{p}\left(U ; \mathbf{R}^{d}\right):= & \left\{u \in G S B V\left(U ; \mathbf{R}^{d}\right): \nabla u \in L^{p}\left(U ; \mathbf{R}^{d \times N}\right)\right. \\
& \text { and } \left.\mathscr{H}^{N-1}\left(S_{u}\right)<+\infty\right\},
\end{aligned}
$$

and if $q \geq 1, G S B V_{q}^{p}\left(U ; \mathbf{R}^{d}\right):=G S B V^{p}\left(U ; \mathbf{R}^{d}\right) \cap L^{q}\left(U ; \mathbf{R}^{d}\right)$. Moreover, we say that a sequence $u_{n} \rightarrow u$ in $G S B V_{q}^{p}\left(U ; \mathbf{R}^{d}\right)$ if $u_{n} \rightarrow u$ a.e. in $U, u_{n} \rightarrow u$ in $L^{q}\left(U ; \mathbf{R}^{d}\right), \nabla u_{n} \rightarrow \nabla u$ in $L^{p}\left(U ; \mathbf{R}^{d \times N}\right)$ and $\mathscr{H}^{N-1}\left(S_{u_{n}}\right)$ is uniformly bounded.

For a given admissible crack $K \in \mathcal{R}\left(\overline{\Omega_{B}^{\varepsilon}}\right)$ and a boundary deformation $\phi \in$ $W^{1, p}\left(\Omega^{\varepsilon} ; \mathbf{R}^{3}\right) \cap L^{q}\left(\Omega^{\varepsilon} ; \mathbf{R}^{3}\right)$, we define the set of admissible deformations with finite energy relative to $(K, \phi)$ by

$$
A D^{\varepsilon}(\phi, K):=\left\{u \in G S B V_{q}^{p}\left(\Omega^{\varepsilon} ; \mathbf{R}^{3}\right): S_{u} \widetilde{\subset} K, u=\phi \mathscr{H}^{2} \text {-a.e. on } \partial_{D} \Omega^{\varepsilon} \backslash K\right\} .
$$

The associate bulk energy is defined by

$$
\mathcal{W}(\varepsilon)(\nabla u):=\int_{\Omega^{\varepsilon}} W(\nabla u(x)) d x,
$$

where $W: \mathbf{R}^{3 \times 3} \rightarrow[0,+\infty)$, the stored energy density, is a quasiconvex function of class $\mathcal{C}^{1}$ satisfying standard $p$-growth and $p$-coercivity conditions $(p>1)$ : there exist $0<\beta^{\prime}<\beta<+\infty$ such that

$$
\beta^{\prime}|\xi|^{p} \leq W(\xi) \leq \beta\left(1+|\xi|^{p}\right) \quad \text { for every } \xi \in \mathbf{R}^{3 \times 3} .
$$


In particular, the functional $\mathcal{W}(\varepsilon): L^{p}\left(\Omega^{\varepsilon} ; \mathbf{R}^{3 \times 3}\right) \rightarrow[0,+\infty)$ defined by

$$
\mathcal{W}(\varepsilon)(\Phi):=\int_{\Omega^{\varepsilon}} W(\Phi(x)) d x
$$

is differentiable on $L^{p}\left(\Omega^{\varepsilon} ; \mathbf{R}^{3 \times 3}\right)$, and its differential $D \mathcal{W}(\varepsilon): L^{p}\left(\Omega^{\varepsilon} ; \mathbf{R}^{3 \times 3}\right) \rightarrow$ $L^{p^{\prime}}\left(\Omega^{\varepsilon} ; \mathbf{R}^{3 \times 3}\right)$, with $p^{\prime}=p /(p-1)$, is given by

$$
\langle D \mathcal{W}(\varepsilon)(\Phi), \Psi\rangle=\int_{\Omega^{\varepsilon}} D W(\Phi(x)): \Psi(x) d x \quad \text { for every } \Phi, \Psi \in L^{p}\left(\Omega^{\varepsilon} ; \mathbf{R}^{3 \times 3}\right) .
$$

On the left-hand side of the previous equality, we have denoted by $\langle\cdot, \cdot\rangle$ the duality pairing between $L^{p}\left(\Omega^{\varepsilon} ; \mathbf{R}^{3 \times 3}\right)$ and $L^{p^{\prime}}\left(\Omega^{\varepsilon} ; \mathbf{R}^{3 \times 3}\right)$.

\subsection{The Forces}

We assume that the body is subjected to the action of conservative body and surface forces with potentials $F$ and $G^{\varepsilon}=\varepsilon G$ respectively. Note that the order of magnitude of the applied forces are exactly those inducing a limiting membrane model (see $[12,13])$.

\subsubsection{The Body Forces}

Let $q \geq 1$, the density of the applied body forces per unit volume at time $t \in[0, T]$ is given by $D_{z} F(t, u(x))$, where $F:[0, T] \times \mathbf{R}^{3} \rightarrow \mathbf{R}$ and the map $z \mapsto F(t, z)$ belongs to $\mathcal{C}^{1}\left(\mathbf{R}^{3}\right)$ for every $t \in[0, T]$. We suppose that for every $t \in[0, T]$, the functional

$$
\mathcal{F}(\varepsilon)(t)(u):=\int_{\Omega^{\varepsilon}} F(t, u(x)) d x
$$

is of class $\mathcal{C}^{1}$ on the space $L^{q}\left(\Omega^{\varepsilon} ; \mathbf{R}^{3}\right)$, and its differential $D \mathcal{F}(\varepsilon)(t): L^{q}\left(\Omega^{\varepsilon} ; \mathbf{R}^{3}\right)$ $\rightarrow L^{q^{\prime}}\left(\Omega^{\varepsilon} ; \mathbf{R}^{3}\right)$, with $q^{\prime}:=q /(q-1)$, is given by

$$
\langle D \mathcal{F}(\varepsilon)(t)(u), v\rangle=\int_{\Omega^{\varepsilon}} D_{z} F(t, u(x)) \cdot v(x) d x \quad \text { for every } u, v \in L^{q}\left(\Omega^{\varepsilon} ; \mathbf{R}^{3}\right) .
$$

We have denoted by $\langle\cdot, \cdot\rangle$ the duality pairing between $L^{q}\left(\Omega^{\varepsilon} ; \mathbf{R}^{3}\right)$ and $L^{q^{\prime}}\left(\Omega^{\varepsilon} ; \mathbf{R}^{3}\right)$.

Concerning the regularity in time, we assume that there exist an exponent $\dot{q}<q$ and, for a.e. $t \in[0, T]$, a functional $\dot{\mathcal{F}}(\varepsilon)(t): L^{\dot{q}}\left(\Omega^{\varepsilon} ; \mathbf{R}^{3}\right) \rightarrow \mathbf{R}$ of class $\mathcal{C}^{1}$, with differential $D \dot{\mathcal{F}}(\varepsilon)(t): L^{\dot{q}}\left(\Omega^{\varepsilon} ; \mathbf{R}^{3}\right) \rightarrow L^{\dot{q}^{\prime}}\left(\Omega^{\varepsilon} ; \mathbf{R}^{3}\right)$, where $\dot{q}^{\prime}=\dot{q} /(\dot{q}-1)$, such that for every $u, v \in L^{q}\left(\Omega^{\varepsilon} ; \mathbf{R}^{3}\right)$, the functions $t \mapsto \dot{\mathcal{F}}(\varepsilon)(t)(u)$ and $t \mapsto$ $\langle D \dot{\mathcal{F}}(\varepsilon)(t)(u), v\rangle$ are integrable on $[0, T]$, and 


$$
\begin{gathered}
\mathcal{F}(\varepsilon)(t)(u)=\mathcal{F}(\varepsilon)(0)(u)+\int_{0}^{t} \dot{\mathcal{F}}(\varepsilon)(s)(u) d s, \\
\langle D \mathcal{F}(\varepsilon)(t)(u), v\rangle=\langle D \mathcal{F}(\varepsilon)(0)(u), v\rangle+\int_{0}^{t}\langle D \dot{\mathcal{F}}(\varepsilon)(s)(u), v\rangle d s
\end{gathered}
$$

for every $t \in[0, T]$. We further assume that $\mathcal{F}(\varepsilon)(t)$ is upper semicontinuous in $L^{q}\left(\Omega^{\varepsilon} ; \mathbf{R}^{3}\right)$ with respect to the pointwise almost everywhere convergence.

Finally, we suppose that $\mathcal{F}(\varepsilon)(t), D \mathcal{F}(\varepsilon)(t), \dot{\mathcal{F}}(\varepsilon)(t)$ and $D \dot{\mathcal{F}}(\varepsilon)(t)$ satisfy suitable $q$-growth conditions: there exist constants $a_{0}>0, a_{1}>0, a_{2}>0, b_{0} \geq 0$, $b_{1} \geq 0, b_{2} \geq 0$, and nonnegative integrable functions on [0,T], $a_{3}, a_{4}, b_{3}$ and $b_{4}$ (uniform in $\varepsilon$ ) such that

$$
\left\{\begin{array}{l}
a_{0}\|u\|_{L^{q}\left(\Omega^{\varepsilon} ; \mathbf{R}^{3}\right)}^{q}-b_{0} \leq-\mathcal{F}(\varepsilon)(t)(u) \leq a_{1}\|u\|_{L^{q}\left(\Omega^{\varepsilon} ; \mathbf{R}^{3}\right)}^{q}+b_{1}, \\
|\langle D \mathcal{F}(\varepsilon)(t)(u), v\rangle| \leq\left(a_{2}\|u\|_{L^{q}\left(\Omega^{\varepsilon} ; \mathbf{R}^{3}\right)}^{q-1}+b_{2}\right)\|v\|_{L^{q}\left(\Omega^{\varepsilon} ; \mathbf{R}^{3}\right)}, \\
|\dot{\mathcal{F}}(\varepsilon)(t)(u)| \leq a_{3}(t)\|u\|_{L^{\dot{q}}\left(\Omega^{\varepsilon} ; \mathbf{R}^{3}\right)}^{\dot{q}}+b_{3}(t), \\
|\langle D \dot{\mathcal{F}}(\varepsilon)(t)(u), v\rangle| \leq\left(a_{4}(t)\|u\|_{L^{\dot{q}}\left(\Omega^{\varepsilon} ; \mathbf{R}^{3}\right)}^{\dot{q}-1}+b_{4}(t)\right)\|v\|_{L^{\dot{q}}\left(\Omega^{\varepsilon} ; \mathbf{R}^{3}\right)} .
\end{array}\right.
$$

\subsubsection{The Surface Forces}

The density of the surface forces on $\partial_{N} \Omega^{\varepsilon}$ at time $t \in[0, T]$, under the deformation $u$ is given by $\varepsilon D_{z} G(t, u(x))$, where $G:[0, T] \times \mathbf{R}^{3} \rightarrow \mathbf{R}$ is such that $z \mapsto G(t, z)$ is of class $\mathcal{C}^{1}\left(\mathbf{R}^{3}\right)$ for every $t \in[0, T]$.

We fix an exponent $r$, related to the trace theorem in Sobolev spaces, such that $r \in[p, p /(3-p)]$ if $p<3$, while $r \geq p$ if $p \geq 3$. We assume that for every $t \in[0, T]$, the functional

$$
\mathcal{G}(\varepsilon)(t)(u):=\varepsilon \int_{\partial_{N} \Omega^{\varepsilon}} G(t, u(x)) d \mathscr{H}^{2}(x)
$$

is of class $\mathcal{C}^{1}$ on $L^{r}\left(\partial_{N} \Omega^{\varepsilon} ; \mathbf{R}^{3}\right)$, with differential $D g(\varepsilon)(t): L^{r}\left(\partial_{N} \Omega^{\varepsilon} ; \mathbf{R}^{3}\right) \rightarrow$ $L^{r^{\prime}}\left(\partial_{N} \Omega^{\varepsilon} ; \mathbf{R}^{3}\right)$, where $r^{\prime}=r /(r-1)$, given by

$$
\begin{gathered}
\langle\mathcal{g}(\varepsilon)(t)(u), v\rangle=\varepsilon \int_{\partial_{N} \Omega^{\varepsilon}} D_{z} G(t, u(x)) \cdot v(x) d \mathscr{H}^{2}(x) \\
\text { for all } u, v \in L^{r}\left(\partial_{N} \Omega^{\varepsilon} ; \mathbf{R}^{3}\right),
\end{gathered}
$$

where $\langle\cdot, \cdot\rangle$ denotes the duality pairing between $L^{r}\left(\partial_{N} \Omega^{\varepsilon} ; \mathbf{R}^{3}\right)$ and $L^{r^{\prime}}\left(\partial_{N} \Omega^{\varepsilon} ; \mathbf{R}^{3}\right)$.

As for the regularity in time, we suppose that for a.e. $t \in[0, T]$, there exists a functional $\dot{g}(\varepsilon)(t): L^{r}\left(\partial_{N} \Omega^{\varepsilon} ; \mathbf{R}^{3}\right) \rightarrow \mathbf{R}$ of class $\mathcal{C}^{1}$, with differential $D \dot{g}(\varepsilon)(t):$ $L^{r}\left(\partial_{N} \Omega^{\varepsilon} ; \mathbf{R}^{3}\right) \rightarrow L^{r^{\prime}}\left(\partial_{N} \Omega^{\varepsilon} ; \mathbf{R}^{3}\right)$, such that for every $u, v \in L^{r}\left(\partial_{N} \Omega^{\varepsilon} ; \mathbf{R}^{3}\right)$, the mappings $t \mapsto \dot{g}(\varepsilon)(t)(u)$ and $t \mapsto\langle D \dot{g}(\varepsilon)(t)(u), v\rangle$ are integrable on [0,T], and 


$$
\begin{gathered}
\mathcal{g}(\varepsilon)(t)(u)=\mathcal{g}(\varepsilon)(0)(u)+\int_{0}^{t} \dot{g}(\varepsilon)(s)(u) d s, \\
\langle D g(\varepsilon)(t)(u), v\rangle=\langle D g(\varepsilon)(0)(u), v\rangle+\int_{0}^{t}\langle D \dot{g}(\varepsilon)(s)(u), v\rangle d s
\end{gathered}
$$

for every $t \in[0, T]$.

Finally, we suppose that $\mathcal{g}(\varepsilon)(t), D \mathcal{g}(\varepsilon)(t), \dot{g}(\varepsilon)(t)$ and $D \dot{g}(\varepsilon)(t)$ satisfy suitable $r$-growth conditions: there exist nonnegative constants $\alpha_{0}, \alpha_{1}, \alpha_{2}, \beta_{0}, \beta_{1}, \beta_{2}$, and nonnegative integrable functions on $[0, T], \alpha_{3}, \alpha_{4}, \beta_{3}$ and $\beta_{4}$ such that

$$
\left\{\begin{array}{l}
-\alpha_{0} \varepsilon\|u\|_{L^{r}\left(\partial_{N} \Omega^{\varepsilon} ; \mathbf{R}^{3}\right)}^{r}-\beta_{0} \varepsilon \leq-\mathcal{g}(\varepsilon)(t)(u) \leq \alpha_{1} \varepsilon\|u\|_{L^{r}\left(\partial_{N} \Omega^{\varepsilon} ; \mathbf{R}^{3}\right)}^{q}+\beta_{1} \varepsilon, \\
|\langle D g(\varepsilon)(t)(u), v\rangle| \leq\left(\alpha_{2} \varepsilon\|u\|_{L^{r}\left(\partial_{N} \Omega^{\varepsilon} ; \mathbf{R}^{3}\right)}^{r-1}+\beta_{2} \varepsilon\right)\|v\|_{L^{r}\left(\partial_{N} \Omega^{\varepsilon} ; \mathbf{R}^{3}\right)}, \\
|\dot{g}(\varepsilon)(t)(u)| \leq \alpha_{3}(t) \varepsilon\|u\|_{L^{r}\left(\partial_{N} \Omega^{\varepsilon} ; \mathbf{R}^{3}\right)}^{r}+\beta_{3}(t) \varepsilon, \\
|\langle D \dot{g}(\varepsilon)(t)(u), v\rangle| \leq\left(\alpha_{4}(t) \varepsilon\|u\|_{L^{r}\left(\partial_{N} \Omega^{\varepsilon} ; \mathbf{R}^{3}\right)}^{r-1}+\beta_{4}(t) \varepsilon\right)\|v\|_{L^{r}\left(\partial_{N} \Omega^{\varepsilon} ; \mathbf{R}^{3}\right)} .
\end{array}\right.
$$

The reason why all the previous coercivity and growth constants/functions are of order $\varepsilon$ is due to the fact that the surface force density $G_{\varepsilon}=\varepsilon G$ of scales like $\varepsilon$.

\subsection{Quasi-Static Evolution}

For a given admissible crack $K \in \mathcal{R}\left(\overline{\Omega_{B}^{\varepsilon}}\right)$ and a given boundary deformation $\phi \in$ $W^{1, p}\left(\Omega^{\varepsilon} ; \mathbf{R}^{3}\right) \cap L^{q}\left(\Omega^{\varepsilon} ; \mathbf{R}^{3}\right)$, the total energy of the configuration $(K, u)$, with $u \in$ $A D^{\varepsilon}(\phi, K)$, at time $t \in[0, T]$ is given by

$$
\mathcal{E}(\varepsilon)(t)(u, K):=\mathcal{W}(\varepsilon)(\nabla u)-\mathcal{F}(\varepsilon)(t)(u)-\mathcal{G}(\varepsilon)(t)(u)+\mathcal{K}(\varepsilon)(K) .
$$

We define a quasi-static evolution with boundary condition $t \mapsto \phi^{\varepsilon}(t)$ as a map $t \mapsto\left(v^{\varepsilon}(t), K^{\varepsilon}(t)\right)$ from $[0, T]$ to $G S B V_{q}^{p}\left(\Omega^{\varepsilon} ; \mathbf{R}^{3}\right) \times \mathcal{R}\left(\overline{\Omega_{B}^{\varepsilon}}\right)$ with the following properties:

(i) Global stability: for all $t \in[0, T]$, we have $v^{\varepsilon}(t) \in A D^{\varepsilon}\left(\phi^{\varepsilon}(t), K^{\varepsilon}(t)\right)$ and

$$
\begin{array}{r}
\mathcal{E}(\varepsilon)(t)\left(v^{\varepsilon}(t), K^{\varepsilon}(t)\right)=\min \left\{\mathcal{E}(\varepsilon)\left(v^{\prime}, K^{\prime}\right): K^{\prime} \in \mathcal{R}\left(\overline{\Omega_{B}^{\varepsilon}}\right), K^{\varepsilon}(t) \widetilde{\subset} K^{\prime}\right. \\
\text { and } \left.v^{\prime} \in A D^{\varepsilon}\left(\phi^{\varepsilon}(t), K^{\prime}\right)\right\} .
\end{array}
$$

(ii) Irreversibility: $K^{\varepsilon}(s) \widetilde{\subset} K^{\varepsilon}(t)$ whenever $s \leq t$.

(iii) Energy balance: the mapping $t \mapsto E(\varepsilon)(t):=\mathcal{E}(\varepsilon)(t)\left(v^{\varepsilon}(t), K^{\varepsilon}(t)\right)$ is absolutely continuous on $[0, T]$ and

$$
\begin{aligned}
\dot{E}(\varepsilon)(t)= & \left\langle D \mathcal{W}(\varepsilon)\left(\nabla v^{\varepsilon}(t)\right), \nabla \dot{\phi}^{\varepsilon}(t)\right\rangle \\
& -\left\langle D \mathcal{F}(\varepsilon)(t)\left(v^{\varepsilon}(t)\right), \dot{\phi}^{\varepsilon}(t)\right\rangle-\dot{\mathcal{F}}(\varepsilon)(t)\left(v^{\varepsilon}(t)\right)
\end{aligned}
$$




$$
-\left\langle D \mathcal{g}(\varepsilon)(t)\left(v^{\varepsilon}(t)\right), \dot{\phi}^{\varepsilon}(t)\right\rangle-\dot{g}(\varepsilon)(t)\left(v^{\varepsilon}(t)\right) .
$$

The following existence result has been proven in [8].

Theorem 1. Let $K_{0}^{\varepsilon} \in \mathcal{R}\left(\overline{\Omega_{B}^{\varepsilon}}\right)$ and $v_{0}^{\varepsilon} \in A D^{\varepsilon}\left(\phi^{\varepsilon}(0), K_{0}^{\varepsilon}\right)$ such that

$$
\mathcal{E}(\varepsilon)(0)\left(v_{0}^{\varepsilon}, K_{0}^{\varepsilon}\right) \leq \mathcal{E}(\varepsilon)(0)\left(v^{\prime}, K^{\prime}\right)
$$

for every $K^{\prime} \in \mathcal{R}\left(\overline{\Omega_{B}^{\varepsilon}}\right)$ with $K_{0}^{\varepsilon} \widetilde{\subset} K^{\prime}$, and every $v^{\prime} \in A D^{\varepsilon}\left(\phi^{\varepsilon}(0), K^{\prime}\right)$. Then there exists a quasi-static evolution $t \mapsto\left(v^{\varepsilon}(t), K^{\varepsilon}(t)\right)$ with boundary deformation $\phi^{\varepsilon}(t)$ such that $\left(v^{\varepsilon}(0), K^{\varepsilon}(0)\right)=\left(v_{0}^{\varepsilon}, K_{0}^{\varepsilon}\right)$.

\section{The Rescaled Configuration}

Our goal is to perform an asymptotic analysis of the quasi-static evolution as the thickness of the film $\varepsilon \rightarrow 0$. As usual in dimension reduction problems (see e.g. $[6,15])$ we rescale the problem into an equivalent one with the advantage of being stated over a fixed domain.

Before doing this we shall make some assumptions on the initial crack. We assume that it is compatible with the geometry of the problem, i.e., that $K_{0}^{\varepsilon}=$ $\gamma_{0} \times(-\varepsilon+\varepsilon \eta, \varepsilon-\varepsilon \eta)$, for some countably $\mathscr{H}^{1}$-rectifiable set $\gamma_{0} \subset \omega$.

We now define $\Omega:=\Omega^{1}, \Omega_{B}:=\Omega_{B}^{1}, \partial_{D} \Omega:=\partial_{D} \Omega^{1}$ and $\partial_{N} \Omega:=\partial_{N} \Omega^{1}$. For $x \in \Omega$, we denote by $x_{\alpha}:=\left(x_{1}, x_{2}\right) \in \omega$ the in-plane variable. We set

$$
\begin{aligned}
\psi^{\varepsilon}\left(t, x_{\alpha}, x_{3}\right) & :=\phi^{\varepsilon}\left(t, x_{\alpha}, \varepsilon x_{3}\right), \\
u_{0}^{\varepsilon}\left(x_{\alpha}, x_{3}\right) & :=v_{0}^{\varepsilon}\left(x_{\alpha}, \varepsilon x_{3}\right), \\
u^{\varepsilon}(t)\left(x_{\alpha}, x_{3}\right) & :=v^{\varepsilon}(t)\left(x_{\alpha}, \varepsilon x_{3}\right), \\
\Gamma^{\varepsilon}(t) & :=\left\{\left(x_{\alpha}, x_{3}\right) \in \overline{\Omega_{B}}:\left(x_{\alpha}, \varepsilon x_{3}\right) \in K^{\varepsilon}(t)\right\} .
\end{aligned}
$$

Changing variables in (1), (2), (4) and (8) leads to

$$
\begin{aligned}
& \mathcal{W}(\varepsilon)\left(\nabla v^{\varepsilon}(t)\right)=\varepsilon \int_{\Omega} W\left(\nabla_{\alpha} u^{\varepsilon}(t) \mid \frac{1}{\varepsilon} \nabla_{3} u^{\varepsilon}(t)\right) d x=: \varepsilon \mathcal{W}^{\varepsilon}\left(\nabla u^{\varepsilon}(t)\right), \\
& \mathcal{K}(\varepsilon)\left(K^{\varepsilon}(t)\right)=\varepsilon \int_{\Gamma^{\varepsilon}(t)}\left|\left(\left(v_{\Gamma^{\varepsilon}(t)}\right)_{\alpha} \mid \frac{1}{\varepsilon}\left(v_{\Gamma^{\varepsilon}(t)}\right)_{3}\right)\right| d \mathscr{H}^{2}=: \varepsilon \mathcal{K}^{\varepsilon}\left(\Gamma^{\varepsilon}(t)\right), \\
& \mathcal{F}(\varepsilon)(t)\left(v^{\varepsilon}(t)\right)=\varepsilon \int_{\Omega} F\left(t, u^{\varepsilon}(t)\right) d x=: \varepsilon \mathcal{F}(t)\left(u^{\varepsilon}(t)\right), \\
& \mathcal{G}(\varepsilon)(t)\left(v^{\varepsilon}(t)\right)=\varepsilon \int_{\partial_{N} \Omega} G\left(t, u^{\varepsilon}(t)\right) d \mathscr{H}^{2}(x)=: \varepsilon \mathcal{g}(t)\left(u^{\varepsilon}(t)\right),
\end{aligned}
$$


where $\nabla_{\alpha}$ (resp. $\left.\nabla_{3}\right)$ denotes the approximate gradient with respect to $x_{\alpha}$ (resp. $x_{3}$ ), and $v_{\Gamma^{\varepsilon}(t)}=\left(\left(v_{\Gamma^{\varepsilon}(t)}\right)_{\alpha},\left(v_{\Gamma^{\varepsilon}(t)}\right)_{3}\right)$ is the normal to $\Gamma^{\varepsilon}(t)$.

As in Section 2.3, we define the functionals $\dot{\mathcal{F}}(t)$ and $\dot{\mathcal{G}}(t)$ so that there hold the analogue of (5), (6), (9) and (10) with $\mathcal{F}, \dot{\mathcal{F}}, g$ and $\dot{g}$ instead of $\mathcal{F}(\varepsilon), \dot{\mathcal{F}}(\varepsilon), \mathcal{g}(\varepsilon)$ and $\dot{g}(\varepsilon)$. Note that $g, \dot{g}, D \mathscr{g}, D \dot{g}, \mathcal{F}, \dot{\mathcal{F}}, D \mathcal{F}$ and $D \dot{\mathcal{F}}$ satisfy analogue growth and coercivity conditions as (7)-(11) with the same exponents, and with coercivity and growth constants/functions independent of $\varepsilon$.

We write $\mathcal{E}^{\varepsilon}(t)$ for the rescaled total energy at time $t$. Then, we deduce that the mapping $t \mapsto\left(u^{\varepsilon}(t), \Gamma^{\varepsilon}(t)\right)$ from $[0, T]$ to $G S B V_{q}^{p}\left(\Omega ; \mathbf{R}^{3}\right) \times \mathcal{R}\left(\overline{\Omega_{B}}\right)$ satisfies the following properties:

(i) Global stability: for all $t \in[0, T]$, we have $u^{\varepsilon}(t) \in A D^{1}\left(\psi^{\varepsilon}(t), \Gamma^{\varepsilon}(t)\right)$ and

$$
\begin{array}{r}
\mathcal{E}^{\varepsilon}(t)\left(u^{\varepsilon}(t), \Gamma^{\varepsilon}(t)\right)=\min \left\{\mathcal{E}^{\varepsilon}\left(u^{\prime}, \Gamma^{\prime}\right): \Gamma^{\prime} \in \mathcal{R}\left(\overline{\Omega_{B}}\right), \Gamma^{\varepsilon}(t) \widetilde{\subset} \Gamma^{\prime}\right. \\
\text { and } \left.u^{\prime} \in A D^{1}\left(\psi^{\varepsilon}(t), \Gamma^{\prime}\right)\right\}
\end{array}
$$

(ii) Irreversibility: $\Gamma^{\varepsilon}(s) \widetilde{\subset} \Gamma^{\varepsilon}(t)$ whenever $s \leq t$.

(iii) Energy balance: the mapping $t \mapsto E^{\varepsilon}(t):=\mathcal{E}^{\varepsilon}(t)\left(u^{\varepsilon}(t), \Gamma^{\varepsilon}(t)\right)$ is absolutely continuous on $[0, T]$ and

$$
\begin{aligned}
\dot{E}^{\varepsilon}(t)= & \left\langle D \mathcal{W}^{\varepsilon}\left(\nabla u^{\varepsilon}(t)\right), \nabla \dot{\psi}^{\varepsilon}(t)\right\rangle \\
& -\left\langle D \mathcal{F}(t)\left(u^{\varepsilon}(t)\right), \dot{\psi}^{\varepsilon}(t)\right\rangle-\dot{\mathcal{F}}(t)\left(u^{\varepsilon}(t)\right) \\
& -\left\langle D \mathcal{G}(t)\left(u^{\varepsilon}(t)\right), \dot{\psi}^{\varepsilon}(t)\right\rangle-\dot{\mathcal{g}}(t)\left(u^{\varepsilon}(t)\right) .
\end{aligned}
$$

\section{Analysis of Static Problem by $\Gamma$-Convergence}

Before going to the study of the quasi-static problem, we will discuss the asymptotic behavior of the total energy as $\varepsilon \rightarrow 0$ thanks to a $\Gamma$-convergence method. Since the work of external forces $\mathcal{F}+g$ corresponds to a continuous perturbation of the sum of the bulk and surface energies, we will not take it into account. We will actually study a weak formulation of the problem replacing the crack by the jump set of the deformation field. Indeed, Let us define $\ell_{\varepsilon}: L^{1}\left(\Omega ; \mathbf{R}^{3}\right) \rightarrow[0,+\infty]$ by

$$
\ell_{\varepsilon}(u):=\int_{\Omega} W\left(\nabla_{\alpha} u \mid \frac{1}{\varepsilon} \nabla_{3} u\right) d x+\int_{S_{u}}\left|\left(\left(v_{u}\right)_{\alpha} \mid \frac{1}{\varepsilon}\left(v_{u}\right)_{3}\right)\right| d \mathscr{H}^{2}
$$

if $u \in G S B V^{p}\left(\Omega ; \mathbf{R}^{3}\right)$, and $+\infty$ if $u \in L^{1}\left(\Omega ; \mathbf{R}^{3}\right) \backslash G S B V^{p}\left(\Omega ; \mathbf{R}^{3}\right)$. Then, the following $\Gamma$-convergence result holds:

Theorem 2. Let $\omega$ be a bounded open subset of $\mathbf{R}^{2}$ and $W: \mathbf{R}^{3 \times 3} \rightarrow \mathbf{R}$ be a continuous function satisfying (3). Then the functional $\ell_{\varepsilon} \Gamma$-converges for the strong $L^{1}\left(\Omega ; \mathbf{R}^{3}\right)$-topology to $\ell: L^{1}\left(\Omega ; \mathbf{R}^{3}\right) \rightarrow[0,+\infty]$ defined by 


$$
\ell(u):=\left\{\begin{array}{cl}
2 \int_{\omega} Q W_{0}\left(\nabla_{\alpha} u\right) d x_{\alpha}+2 \mathscr{H}^{1}\left(S_{u}\right) & \text { if } u \in G S B V^{p}\left(\omega ; \mathbf{R}^{3}\right), \\
+\infty & \text { otherwise }
\end{array}\right.
$$

where $W_{0}(\bar{\xi}):=\inf \left\{W(\bar{\xi} \mid z): z \in \mathbf{R}^{3}\right\}$ for every $\bar{\xi} \in \mathbf{R}^{3 \times 2}$, and $Q W_{0}$ is the quasiconvexification of $W_{0}$.

This result has been proven in [2] (see also [3]) in a $S B V^{p}$ framework, and one can notice that much easier arguments lead to the analogue in $G S B V^{p}$ as stated in Theorem 2. Indeed, in $G S B V^{p}$, there is no lack of compactness and it is not necessary to appeal to a truncation argument as in [2, lemma 3.3]. It follows immediately from the $G S B V$-compactness theorem [1, theorem 4.36] that any minimizing sequence $\left(u_{\varepsilon}\right) \subset G S B V^{p}\left(\Omega ; \mathbf{R}^{3}\right)$ with uniformly bounded energy is relatively compact in $G S B V^{p}\left(\Omega ; \mathbf{R}^{3}\right.$ ), and that any accumulation point is independent of $x_{3}$ (we identify those functions to $\left.G S B V^{p}\left(\omega ; \mathbf{R}^{3}\right)\right)$. The proof of the lower bound is exactly the same than [2, lemma 3.9] using the lower semicontinuity result in $G S B V^{p}$ (see e.g. [8, theorem 2.8]). The construction of a recovery $\left(\bar{u}_{\varepsilon}\right)$ can be performed as in [15]: it suffices to take $\bar{u}_{\varepsilon}\left(x_{\alpha}, x_{3}\right):=u\left(x_{\alpha}\right)+\varepsilon x_{3} b_{\varepsilon}\left(x_{\alpha}\right)$ for some suitable function $b_{\varepsilon} \in \mathcal{C}_{c}^{\infty}\left(\omega ; \mathbf{R}^{3}\right)$, and then we appeal to a classical relaxation result in $G S B V^{p}$. We also refer to [5] for an alternative proof using a singular perturbation argument.

\section{Analysis of the Quasi-Static Problem}

In view of Theorem 2, one can guess that the 3D quasi-static evolution - whose existence is ensured by Theorem 1 - will converge in a certain sense to a $2 \mathrm{D}$ quasistatic evolution associated to the $\Gamma$-limit model.

We assume that $\psi^{\varepsilon} \rightarrow \psi$ in $W^{1,1}\left([0, T] ; W^{1, p}\left(\Omega ; \mathbf{R}^{3}\right) \cap L^{q}\left(\Omega ; \mathbf{R}^{3}\right)\right)$ and that the sequence $\left((1 / \varepsilon) \nabla_{3} \psi^{\varepsilon}\right)$ is strongly converging in $W^{1,1}\left([0, T] ; L^{p}\left(\Omega ; \mathbf{R}^{3}\right)\right)$. In particular, the limit function $\psi \in W^{1,1}\left([0, T] ; W^{1, p}\left(\omega ; \mathbf{R}^{3}\right) \cap L^{q}\left(\omega ; \mathbf{R}^{3}\right)\right)$ is independent of $x_{3}$.

We first derive some compactness of $\left(u^{\varepsilon}(t), \Gamma^{\varepsilon}(t)\right)$. Indeed taking $\left(\psi^{\varepsilon}(t), \Gamma^{\varepsilon}(t)\right)$ as competitor in the minimality, and using the growth an coercivity properties satisfied by the functionals $\mathcal{W}_{\varepsilon}, \mathcal{F}$ and $g$ implies that the sequence of approximate scaled gradients $\left(\nabla_{\alpha} u^{\varepsilon}(t) \mid(1 / \varepsilon) \nabla_{3} u^{\varepsilon}(t)\right)$ is bounded in $L^{p}\left(\Omega ; \mathbf{R}^{3 \times 3}\right)$, and the sequence $\left(u^{\varepsilon}(t)\right)$ is bounded in $L^{\dot{q}}\left(\Omega ; \mathbf{R}^{3}\right) \cap L^{q}\left(\Omega ; \mathbf{R}^{3}\right)$. Moreover, since $u^{\varepsilon}(t) \in$ $W^{1, p}\left(\Omega \backslash \overline{\Omega_{B}} ; \mathbf{R}^{3}\right)$, the trace theorem and the choice of the exponent $r$ ensures that $\left(u^{\varepsilon}(t)\right)$ is compact in $L^{r}\left(\partial_{N} \Omega ; \mathbf{R}^{3}\right)$. Then we use the energy balance together with the growth and coercivity conditions satisfied by $D \mathcal{F}(t), D g(t), \dot{\mathcal{F}}(t)$ and $\dot{g}(t)$ to ensure that

$$
\sup _{\varepsilon>0} \int_{\Gamma^{\varepsilon}(t)}\left|\left(\left(\nu_{\Gamma^{\varepsilon}(t)}\right)_{\alpha} \mid \frac{1}{\varepsilon}\left(v_{\Gamma^{\varepsilon}(t)}\right)_{3}\right)\right| d \mathcal{H}^{2}<+\infty .
$$

At this step, we are in position to use a mean convergence for rectifiable sets introduced in [2], very close to the $\sigma^{p}$-convergence in [8,9]: 
Definition 1. Let $\varepsilon_{n} \searrow 0^{+}, \Gamma_{n} \subset \Omega$ be a sequence of countably $\mathscr{H}^{2}$-rectifiable sets, and $\gamma \subset \omega$ be a countably $\mathscr{H}^{1}$-rectifiable set. We say that $\Gamma_{n}$ converges to $\gamma$ in $\Omega$ if

$$
\int_{\Gamma_{n}}\left|\left(\left(v_{\Gamma_{n}}\right)_{\alpha} \mid \frac{1}{\varepsilon_{n}}\left(v_{\Gamma_{n}}\right)_{3}\right)\right| d \mathscr{H}^{2} \leq C,
$$

and the following properties hold:

(a) if $u_{k} \rightarrow u$ in $S B V^{p}(\Omega), S_{u_{k}} \widetilde{\subset} \Gamma_{n_{k}}$ and $\int_{\Omega}\left|\left(\nabla_{\alpha} u_{k} \mid\left(1 / \varepsilon_{n_{k}}\right) \nabla_{3} u_{k}\right)\right|^{p} d x \leq C$, for some subsequence $\left(\varepsilon_{n_{k}}\right) \subset\left(\varepsilon_{n}\right)$, then $u \in S B V^{p}(\omega)$ and $S_{u} \widetilde{\subset} \gamma$;

(b) there exist a function $u \in S B V^{p}(\omega)$ and a sequence $\left(u_{n}\right) \subset S B V^{p}(\Omega)$ such that $u_{n} \rightarrow u$ in $S B V^{p}(\Omega), S_{u_{n}} \widetilde{\subset} \Gamma_{n}, \int_{\Omega}\left|\left(\nabla_{\alpha} u_{n} \mid\left(1 / \varepsilon_{n}\right) \nabla_{3} u_{n}\right)\right|^{p} d x \leq C$, and $S_{u} \cong \gamma$.

Then, using [2, proposition 4.3], one can extract a subsequence $\left(\varepsilon_{n}\right)$ (independently of $t$ ) and find a countably $\mathscr{H}^{1}$-rectifiable set $\gamma(t)$ increasing with respect to $t$ such that $\Gamma^{\varepsilon_{n}}(t)$ converges to $\gamma(t)$ in sense of Definition 1. Note that it is possible to prove that $\gamma(0)=\gamma_{0}$. Moreover the estimates we have on the sequence $\left(u^{\varepsilon_{n}}(t)\right)$ allow us to apply the $G S B V$-Compactness Theorem (see [1, theorem 4.36]) which ensures, for each $t \in[0, T]$, the existence of a $t$-dependent subsequence $\left(\varepsilon_{n_{t}}\right) \subset$ $\left(\varepsilon_{n}\right)$ such that $u^{\varepsilon_{n}}(t) \rightarrow u(t)$ in $G S B V_{q}^{p}\left(\Omega ; \mathbf{R}^{3}\right)$. Moreover the limit deformation $u \in G S B V_{q}^{p}\left(\omega ; \mathbf{R}^{3}\right)$, i.e., it is independent of $x_{3}$.

We claim that the pair $(u(t), \gamma(t))$ is a quasi-static evolution associated to the $\Gamma$ limit model. We already proved the irreversibility condition. To show the minimality property we use the following jump transfer theorem whose proof can be obtained exactly as in [2, theorem 4.4], using [8, theorem 5.3] instead of [11, theorem 2.1]. Let $\omega^{\prime} \subset \mathbf{R}^{2}$ a bounded open set containing $\bar{\omega}$, and define $\Omega^{\prime}:=\omega^{\prime} \times(-1,1)$.

Theorem 3. Let $\Gamma_{n} \in \mathcal{R}\left(\overline{\Omega_{B}}\right)$ be a sequence of countably $\mathscr{H}^{2}$-rectifiable sets converging to $\gamma$ in the sense of Definition 1. Then, for every $v \in G S B V_{q}^{p}\left(\omega^{\prime} ; \mathbf{R}^{3}\right)$, there exists a sequence $\left(v_{n}\right) \subset G S B V_{q}^{p}\left(\Omega^{\prime} ; \mathbf{R}^{3}\right)$ such that $v_{n}=v$ a.e. on $\Omega^{\prime} \backslash \bar{\Omega}$,

- $\quad v_{n} \rightarrow v$ in $L^{q}\left(\Omega^{\prime} ; \mathbf{R}^{3}\right)$,

- $\quad\left(\nabla_{\alpha} v_{n} \mid \frac{1}{\varepsilon_{n}} \nabla_{3} v_{n}\right) \rightarrow\left(\nabla_{\alpha} v \mid 0\right)$ in $L^{p}\left(\Omega^{\prime} ; \mathbf{R}^{3 \times 3}\right)$,

- $\limsup _{n \rightarrow+\infty} \int_{S_{v_{n} \backslash \Gamma_{n}}}\left|\left(\left(v_{v_{n}}\right)_{\alpha} \mid \frac{1}{\varepsilon_{n}}\left(v_{v_{n}}\right)_{3}\right)\right| d \mathscr{H}^{2} \leq 2 \mathscr{H}^{1}\left(S_{v} \backslash \gamma\right)$.

Arguing exactly as in the proof of [2, lemma 5.5] and using the upper semicontinuity property of $\mathcal{F}(t)$ together with the continuity of $g(t)$ (which comes from the trace theorem in $W^{1, p}$ and the choice of the exponent $r$ ), one can show that for every $t \in[0, T], u(t)$ minimizes

$$
v \mapsto 2 \int_{\omega} Q W_{0}\left(\nabla_{\alpha} v\right) d x_{\alpha}+2 \mathcal{H}^{1}\left(S_{v} \backslash \gamma(t)\right)-2 \mathcal{F}(t)(v)-2 \mathscr{g}(t)(v),
$$

among $\left\{v \in G S B V_{q}^{p}\left(\omega ; \mathbf{R}^{3}\right): v=\psi(t) \mathscr{H}^{1}\right.$-a.e. on $\left.\partial \omega\right\}$. Moreover one has convergence of the bulk energy (for the sequence $\varepsilon_{n}$ ) 


$$
\int_{\Omega} W\left(\nabla_{\alpha} u^{\varepsilon_{n}}(t) \mid \frac{1}{\varepsilon_{n}} \nabla_{3} u^{\varepsilon_{n}}(t)\right) d x \rightarrow 2 \int_{\omega} Q W_{0}\left(\nabla_{\alpha} u(t)\right) d x_{\alpha},
$$

as well as weak convergence of the stress (for the subsequence $\varepsilon_{n_{t}}$ )

$$
D W\left(\nabla_{\alpha} u^{\varepsilon_{n_{t}}}(t) \mid \frac{1}{\varepsilon_{n_{t}}} \nabla_{3} u^{\varepsilon_{n_{t}}}(t)\right) \rightarrow\left(D\left(Q W_{0}\right)\left(\nabla_{\alpha} u(t) \mid 0\right)\right) \quad \text { in } L^{p^{\prime}}\left(\Omega ; \mathbf{R}^{3 \times 3}\right)
$$

at every time. Remark that by [2, proposition 4.7], the function $Q W_{0}$ is of class $\mathcal{C}^{1}$. For every $v \in G S B V_{q}^{p}\left(\omega ; \mathbf{R}^{3}\right)$ such that $v=\psi(t) \mathscr{H}^{1}$-a.e. on $\partial \omega$, and every countably $\mathscr{H}^{1}$-rectifiable set $\gamma \subset \omega$, we define

$$
\mathcal{E}(t)(v, \gamma)=2 \int_{\omega} Q W_{0}\left(\nabla_{\alpha} v\right) d x_{\alpha}+2 \mathscr{H}^{1}(\gamma)-2 \mathcal{F}(t)(v)-2 g(t)(v) .
$$

The minimality property proven above exactly says that

$$
\begin{aligned}
\mathscr{E}(t)(u(t), \gamma(t))= & \min \left\{\mathcal{E}(t)(v, \gamma): \gamma \text { countably } \mathscr{H}^{1}-\text { rectifiable set in } \omega,\right. \\
& \left.v \in G S B V_{q}^{p}\left(\omega ; \mathbf{R}^{3}\right) \text { such that } v=\psi(t) \mathscr{H}^{1} \text {-a.e. on } \partial \omega\right\}
\end{aligned}
$$

(see e.g. [2, remark 5.4]). Moreover, since we have reduced dimension, the functional $\mathcal{g}(t)$ becomes a bulk force as well as $\mathcal{F}(t)$.

It remains to prove the energy balance. Arguing word for word as in [8], approximating Bochner integrals by suitable Riemann sums, one can show that

$$
\begin{aligned}
\mathscr{E}(t)(u(t), \gamma(t)) \geq & \&(0)(u(0), \gamma(0))+2 \int_{0}^{t}\left(\left\langle D W_{0}\left(\nabla_{\alpha} u(s)\right), \nabla_{\alpha} \dot{\psi}(s)\right)\right\rangle \\
& -\langle D \mathcal{F}(t)(u(s)), \dot{\psi}(s)\rangle-\dot{\mathcal{F}}(s)(u(s)) \\
& -\langle D g(s)(u(s)), \dot{\psi}(s)\rangle-\dot{\mathcal{g}}(s)(u(s))) d s,
\end{aligned}
$$

where $\mathcal{W}_{0}: L^{p}\left(\omega ; \mathbf{R}^{3 \times 2}\right) \rightarrow[0,+\infty)$ is defined by $\mathcal{W}_{0}(\Phi):=$ $\int_{\omega} Q W_{0}(\Phi(x)) d x_{\alpha}$. By [2, Proposition 4.7], we deduce that $W_{0}$ is of class $\mathcal{C}^{1}$ with differential $D W_{0}: L^{p}\left(\omega ; \mathbf{R}^{3 \times 2}\right) \rightarrow L^{p^{\prime}}\left(\omega ; \mathbf{R}^{3 \times 2}\right)$ given by

$$
\left\langle D W_{0}(\Phi), \Psi\right\rangle=\int_{\omega} D\left(Q W_{0}\right)(\Phi(x)): \Psi(x) d x_{\alpha} .
$$

We prove the other inequality exactly as in [2, lemma 5.8], using the upper semicontinuity property of the functional $\mathcal{F}(t)$ and the weak convergence of the stresses (12) already mentioned above. We also deduce the convergence of the surface en$\operatorname{ergy}$ (for the sequence $\varepsilon_{n}$ ):

$$
\int_{\Gamma^{\varepsilon_{n}(t)}}\left|\left(\left(v_{\Gamma^{\varepsilon_{n}}(t)}\right)_{\alpha} \mid \frac{1}{\varepsilon_{n}}\left(v_{\Gamma^{\varepsilon_{n}}(t)}\right)_{3}\right)\right| d \mathscr{H}^{2} \rightarrow 2 \mathscr{H}^{1}(\gamma(t)) .
$$


In conclusion, we have proven the following result which states that any $3 \mathrm{D}$ quasistatic crack evolution converges to a $2 \mathrm{D}$ quasi-static evolution associated to the $\Gamma$ limit model:

Theorem 4. There exist a two-dimensional quasi-static evolution $t \mapsto(u(t), \gamma(t))$ relative to the boundary data $\psi(t)$ for the $\Gamma$-limit model, and a sequence $\varepsilon_{n} \searrow 0^{+}$ such that for any $t \in[0, T]$,

- $\quad \Gamma^{\varepsilon_{n}}(t)$ converges to $\gamma(t)$ in sense of Definition 1 ;

- $u^{\varepsilon_{n_{t}}}(t) \rightarrow u(t)$ in $G S B V_{q}^{p}\left(\Omega ; \mathbf{R}^{3}\right)$ for some $t$-dependent subsequence $\left(\varepsilon_{n_{t}}\right) \subset$ $\left(\varepsilon_{n}\right)$;

- $\quad$ the total energy $E^{\varepsilon_{n}}(t)$ converges to $\&(t)$, and more precisely

$$
\begin{gathered}
\int_{\Omega} W\left(\nabla_{\alpha} u^{\varepsilon_{n}}(t) \mid \frac{1}{\varepsilon_{n}} \nabla_{3} u^{\varepsilon_{n}}(t)\right) d x \rightarrow 2 \int_{\omega} Q W_{0}\left(\nabla_{\alpha} u(t)\right) d x_{\alpha}, \\
\int_{\Gamma^{\varepsilon_{n}}(t)}\left|\left(\left(v_{\Gamma^{\varepsilon_{n}}(t)}\right)_{\alpha} \mid \frac{1}{\varepsilon_{n}}\left(\nu_{\Gamma^{\varepsilon_{n}}(t)}\right)_{3}\right)\right| d \mathscr{H}^{2} \rightarrow 2 \mathscr{H}^{1}(\gamma(t)) .
\end{gathered}
$$

\section{Acknowledgement}

The research of the author has been supported by the Chair "Mathematical Modelling and Numerical Simulation, F-EADS Ecole Polytechnique INRIA F-X".

\section{References}

1. L. Ambrosio, N. Fusco and D. Pallara, Functions of Bounded Variation and Free Discontinuity Problems, Oxford University Press, Oxford, 2000.

2. J.-F. Babadjian, Quasistatic evolution of a brittle thin film, Calc. Var. and PDEs 26(1), 2006, 69-118.

3. J.-F. Babadjian: Lower semicontinuity of quasiconvex bulk energies in $S B V$ and integral representation in dimension reduction, SIAM J. Math. Anal. 39(6), 2008, 1921-1950.

4. B. Bourdin, G.A. Francfort and J.-J. Marigo: The Variational Approach to Fracture, Springer, Amsterdam, 2008.

5. A. Braides and I. Fonseca: Brittle thin films, Appl. Math. Optim. 44, 2001, 299-323.

6. A. Braides, I. Fonseca and G.A. Francfort: 3D-2D Asymptotic analysis for inhomogeneous thin films, Indiana Univ. Math. J. 49, 2000, 1367-1404.

7. G. Dal Maso: An Introduction to $\Gamma$-Convergence, Birkhäuser, Boston, 1993.

8. G. Dal Maso, G.A. Francfort and R. Toader: Quasi-static crack growth in nonlinear elasticity, Arch. Rational Mech. Anal. 176, 2005, 165-225.

9. G. Dal Maso, G.A. Francfort and R. Toader: Quasi-static evolution in brittle fracture: The case of bounded solutions, Calculus of Variations: Topics from the Mathematical Heritage of E. De Giorgi. Quaderni di Matematica 14, 2005, 247-265.

10. G.A. Francfort and J.-J. Marigo: Revisiting brittle fracture as an energy minimization problem, J. Mech. Phys. Solids 46, 1998, 1319-1342. 
11. G.A. Francfort and G.J. Larsen: Existence and convergence for quasi-static evolution in brittle fracture, Comm. Pure Appl. Math. 56, 2003, 1465-1500.

12. G. Friesecke, R. James and S. Müller: A hierarchy of plate models derived from nonlinear elasticity by $\Gamma$-convergence, Arch. Rational Mech. Anal. 180(2), 2006, 183-236.

13. D. Fox, A. Raoult and J.C. Simo: A justification of nonlinear properly invariant plate theories, Arch. Rational. Mech. Anal. 25, 1992, 157-199.

14. A. Giacomini and M. Ponsiglione: A $\Gamma$-convergence approach to stability of unilateral minimality properties in fracture mechanics and applications, Arch. Rational Mech. Anal. 180, 2006, 399-447.

15. H. Le Dret and A. Raoult: The nonlinear membrane model as variational limit of nonlinear three-dimensional elasticity, J.Math. Pures Appl. 74, 1995, 549-578.

16. A. Mielke, T. Roubicek and U. Stefanelli: $\Gamma$-limits and relaxations for rate-independent evolutionary problems, Calc. Var. and PDEs 31, 2008, 387-416. 